Copyright C1997, American Institute of Aeronautics and Astronautics, Inc.

AIAA Meeting Papers on Disc, January 1997

A9715379, AIAA Paper 97-0308

\title{
MSX design driven by targets and backgrounds
}

\section{John D. Mill}

Michigan, Environmental Research Inst., Ann Arbor

John T. Stair, Jr.

Visidyne, Inc., Burlington, $M A$

\section{AIAA, Aerospace Sciences Meeting \& Exhibit, 35th, Reno, NV, Jan. 6-9, 1997}

\begin{abstract}
A suite of state-of-the-art sensors, including a cryogenic infrared scanning radiometer and a Fourier-transform spectrometer, several visible and ultraviolet imagers and spectrographic imagers, and a set of contamination monitoring instruments, integrated with a highly capable spacecraft, was launched into a polar orbit on April 24, 1996. The optical sensors cover the spectrum from the far ultraviolet through the very-longwave infrared (110 nm to 28 microns). The Midcourse Space Experiment (MSX) satellite, funded and managed by the Ballistic Missile Defense Organization (BMDO), is a long-duration, observatory style measurement platform that will collect almost two terabytes of high-quality data on Earth, earthlimb, and celestial backgrounds, ICBM-style targets and resident space objects. The requirements for demonstrating space-based surveillance, acquisition, tracking, and discrimination of ballistic reentry vehicles and penetration aids form the basis for the design of the MSX satellite and sensors. MSX is presently performing four data collection events per day, principally in the areas of Earth, earthlimb, and celestial backgrounds. Successful target measurements have been performed on several ballistic launches. A large number of successful space surveillance experiments have been performed. This paper summarizes the goals and design of the spacecraft and sensors. (Author)
\end{abstract}


AIAA-97-0308

\title{
MSX DESIGN DRIVEN BY TARGETS AND BACKGROUNDS
}

\author{
John D. Mill \\ MSX Project Scientist \\ Environmental Research Institute of Michigan \\ Arlington, Virginia \\ A.T. Stair, Jr. \\ MSX Chief Scientist \\ Visidyne, Inc. \\ Burlington, Massachusetts
}

\begin{abstract}
A suite of state-of-the-art sensors, including a cryogenic infrared scanning radiometer and Fourier-transform spectrometer, several visible and ultraviolet imagers and spectrographic imagers, and a set of contamination monitoring instruments, integrated with a highly capable spacecraft, was launched into a polar orbit on April 24, 1996. The optical sensors cover the spectrum from the far ultraviolet through the very-longwave infrared $(110 \mathrm{~nm}$ to $28 \mu \mathrm{m})$. The Midcourse Space Experiment (MSX) satellite, funded and managed by the Ballistic Missile Defense Organization (BMDO), is a long-duration, "observatory" style measurement platform that will collect almost two terabytes of high-quality data on Earth, earthlimb, and celestial backgrounds, ICBMstyle targets and resident space objects. The requirements for demonstrating space-based surveillance, acquisition, tracking, and discrimination of ballistic reentry vehicles and penetration aids form the basis for the design of the MSX satellite and sensors. MSX is presently performing four data collection events per day, principally in the areas of earth, earthlimb, and celestial backgrounds. Successful target measurements have been performed on several
\end{abstract}

ballistic launches. A large number of successful space surveillance experiments have been performed. This paper summarizes the goals and design of the spacecraft and sensors.

\section{Introduction}

The functions of BMDO midcourse (the phase of missile flight between boost and reentry) sensor systems are to detect, acquire, and track targets and to discriminate lethal from nonlethal objects. To perform these functions, the design of a surveillance system must incorporate a full understanding of the behavior and resulting signatures of the expected targets and the effects of the backgrounds against which these targets must be viewed. This in turn requires detailed characterization and careful modeling of potential target objects and their associated phenomenology, and high-fidelity models of the terrestrial, earthlimb and celestial backgrounds. These data and models are required across the optical spectrum, over the entire globe, and for an extended time period to account for seasonal and geographic variations.

The BMDO has developed a suite of phenomenology models to address these issues, such as the Optical Signature Code (OSC) and 
the Strategic High Altitude Radiance Code (SHARC) ${ }^{1}$. These models have been incorporated into a framework code called the Strategic Scene Generator Model (SSGM) ${ }^{2}$, which provides validated scenes at the resolution, wavelength, frame rate, and field-of-view of a candidate sensor under development or test. Although past experiments have returned valuable data, they were of short duration or limited resolution or sensitivity. MSX is designed to address these limitations and provide the necessary data sets over the globe, during all seasons, and at the resolution and sensitivity necessary to address system issues.

MSX was created primarily for infrared data collection and as a functional demonstration of the Spatial Infrared Imaging Telescope III (SPIRIT III) cryogenic sensor. It evolved from the national Midcourse Sensor Study (MSS) sponsored by the Strategic Defense Initiative Organization (SDIO, now the Ballistic Missile Defense Organization) and the Air Force in 1987. A major finding of that study was that space surveillance, tracking, and discrimination functions did not necessarily require the large one meter diameter mirrors for the infrared sensors, which had been until then a nominal design parameter. As a result of the MSS, SPIRIT III was designed with the support of a broad aerospace community, including the U. S. Air Force, U. S. Army, SDIO, federally funded research and development centers, and contractors, and has only a 35-cm aperture. The issue of mirror size is critical since, historically, satellite mass and therefore costs tend to scale with mirror diameter to the 2.6 power for spaceborne electro-optical sensors. This article examines some unique DoD technical issues that will be addressed by MSX and, in particular, by the SPIRIT III infrared radiometric sensor, which was built and calibrated by the Space Dynamics Laboratory of Utah State University.
The primary purpose of MSX is to collect and analyze target and background phenomenology data to address BMDO midcourse sensor requirements. MSX will demonstrate midcourse sensor functions from space, collect midcourse target and background databases, and demonstrate critical sensor technologies. ${ }^{3}$

\section{Target Functional Demonstrations and Phenomenology}

The MSX Early Midcourse and Theater Midcourse Principal Investigator teams are carrying out functional demonstrations and collecting phenomenology data on well-characterized midcourse targets. Functional demonstrations using these data will establish the ability of a space-based sensor to acquire and track threat objects in the presence of real backgrounds using current optical sensor technology. Target phenomenology data will be used to validate target signature models, such as the Optical Signatures Code (OSC).

\section{Demonstrations of Acquisition and Tracking}

The early midcourse phase of a ballistic missile engagement begins with the release of the post-boost vehicle (PBV). Subsequent activities include maneuvers to position the reentry vehicles (RV) for proper targeting, plus any activities associated with target deployment, such as spin-up and release. The late midcourse phase begins when the target complex is still seen as a cluster of closely-spaced objects (CSO) but is beginning to evolve into individually resolved objects. In either phase, the PBV and RVs may use techniques to confuse the surveillance sensors, including, for example, the deployment of decoys that mimic warheads, 
traffic balloons to confuse tracking and discrimination sensors, and chaff. In addition, various design elements of the PBV or RVs may be intended to alter or eliminate signature features which could aid in discrimination.

The primary focus of the early midcourse target experiments is the resolution of issues in CSO tracking and evolution, metric and radiometric discrimination, deployment phenomenology, and PBV tracking. These experiments will acquire the first extensive database of early midcourse target signature phenomenology observed from a space-based sensor.

The cooperative targets (CT) experiments team address issues of acquisition, tracking, data fusion, and discrimination for the midcourse phase of both strategic and theater ballistic missile defense engagements. In addition, unique data are to be obtained on the behavior of long wave infrared and visible target signatures which permit the detailed correlation of infrared signatures and their phenomenology with both body rotational dynamics and dynamics evolution.

\section{Target Signature Data Collection}

The MSX target signature measurement requirements are addressed in three dedicated MSX strategic missile flights, and several cooperative strategic and theater flights. The MSX Early Midcourse dedicated flight on 31 August 1996 employed the Operational Deployment Experiment Simulator (ODES) PBV to deploy a total of 26 midcourse objects of various types under differing deployment conditions. Cooperative missions to date include the Theater Countermeasures Mitigation Program (TCMP) 2b flight on 15 July from Kwajalein Missile Range, the test of the Multi-Service Launch System
(MSLS) on a Minuteman II out of Vandenberg AFB on 27 September, and the Red Tigress III launch from Wallops Island on 16 October.

During all dedicated and cooperative target flights, the objects are viewed simultaneously by the MSX satellite and a number of aircraft-, ship-, and ground-based sensors to establish ground-truth and to provide data for sensor-to-sensor correlation studies. Other experiments will acquire background data in target track mode with realistic engagements, to investigate acquisition and tracking functions under a variety of stressing (spatially complex, or cluttered) background conditions.

\section{Targets-related SPIRIT III Design Issues}

Very sensitive infrared sensors are required to detect and track ballistic reentry vehicles and associated objects, day or night. Such sensors must operate in wavelength regions where these hard bodies radiate energy or reflect the Earth's radiant energy. For a $300 \mathrm{~K}$ gray body, $85 \%$ of the energy falls between 6 and 30 $\mathrm{mm}$. The detection/tracking problem for sunlit objects can be achieved by using simpler visible sensors, which will be demonstrated by the MSX visible sensor suite. Many parameters enter into optimizing the number of satellites and constellations, the size of sensor aperture, wavelength selection, etc. In particular, these issues depend on the definition of the threat (i.e., number of threat objects and penetration aids, deployment, size, shape, temperature, emissivity, etc.). For demonstration purposes only (since MSX is an experiment rather than a prototype), the $900-\mathrm{km}$ orbit and $35-\mathrm{cm}$ diameter of the SPIRIT III primary mirror, with a 90 mrad pixel instantaneous field of view, will be used for subsequent discussions. 
Table 1. SPIRIT III Radiometer Passbands and Calibrated Sensitivities

\begin{tabular}{|c|c|c|c|c|}
\hline \multirow[b]{3}{*}{ Band } & \multirow{3}{*}{$\begin{array}{c}\text { Passband at nominal } \\
50 \% \text { transmission } \\
(\mu \mathrm{m})\end{array}$} & \multicolumn{3}{|c|}{ Noise equivalent flux densities in-band } \\
\hline & & \multicolumn{2}{|c|}{ Integration time } & \multirow{2}{*}{$\begin{array}{c}\text { With TDl } \\
2.7 \mathrm{~ms}\end{array}$} \\
\hline & & $2.7 \mathrm{~ms}$ & $14 \mathrm{~ms}$ & \\
\hline A & $6.0-10.9$ & 2.5 & 0.5 & 0.6 \\
\hline B1 & $4.22-4.36$ & 21 & 4.5 & 6.6 \\
\hline B2 & $4.24-4.45$ & 18 & 3.9 & 5.7 \\
\hline $\mathbf{C}$ & $11.1-13.2$ & 8.6 & 1.8 & 1.6 \\
\hline $\mathbf{D}$ & $13.5-16.0$ & 5.1 & 1.1 & 2.0 \\
\hline $\mathbf{E}$ & $18.3-26.0$ & 17 & 3.5 & 11 \\
\hline
\end{tabular}

The spectral passbands and sensitivities of the SPIRIT III radiometer are given in Table 1. These calibrated values for the noise equivalent flux densities were obtained before the most recent refurbishment of the sensor and may vary somewhat from actual flight values. Bands A, C, $D$, and $E$ are separate filtered focal plane arrays; bands B1 and B2 are implemented by filtering separate halves of one focal plane array. Bands $\mathrm{B} 1$ and $\mathrm{B} 2$ are centered in the optically thick $\boldsymbol{V}_{3}$ band of $\mathrm{CO}_{2}$ for measurements looking at the hard Earth (below the horizon). All other bands, designed for viewing into space (above the horizon), accept so much radiation that their detectors saturate below tangent heights of 40 to $60 \mathrm{~km}$, owing to atmospheric limb radiance ${ }^{4}$.

Perhaps the most critical question for space-borne infrared sensor performance concerns range. Assume a nominal ballistic reentry vehicle to be a cone of about one meter in length with a 0.5 meter base diameter, an emissivity of 0.5 and a temperature of $300 \mathrm{~K}$. Depending on the viewing angle, the projected area varies from about 0.2 to $0.8 \mathrm{~m}^{2}$. Using $0.3 \mathrm{~m}^{2}$ for the pro- jected area, the total radiant signal is $22 \mathrm{~W} / \mathrm{sr}$. For a $300 \mathrm{~K}$ blackbody, about $30 \%$ of the emitted energy falls into band $A$ ( 6 to $11 \mu \mathrm{m}$ ), resulting in a $6.6 \mathrm{~W} / \mathrm{sr}$ signal in this band. At a range of $6400 \mathrm{~km}$, the target flux (irradiance) at the aperture of SPIRIT III in band A is $16 \cdot 10^{-18}$ $\mathrm{W} / \mathrm{cm} 2$. From Table 1 , using the noise equivalent flux density for a point source (with TDI) of $6 \cdot 10^{-19} \mathrm{~W} / \mathrm{cm}^{2}$, this scenario would yield a measurement (independent of backgrounds) with a signal-to-noise ratio of about 25 .

Other important target-related "global" parameters for an infrared sensor are the total field of view, the instantaneous pixel resolution, and scene revisit time. The SPIRIT III field-ofregard has a one-degree "vertical" extent, defined by the height of the column of detectors. There are 5 focal plane arrays (chips) of up to 8 columns of detectors each with 192 detectors per column. An internal scanning mirror sweeps the vertical field of view of the radiometer over a wider field of regard and can be commanded into any one of three scan modes of $3.0,1.5$, or $0.75^{\circ}$ nominal total "horizontal" angular extent. The mirror scans at a constant rate of $0.46 \mathrm{deg} / \mathrm{s}$ 
in object space, producing average revisit times of $7.17,3.59$, and $1.79 \mathrm{~s}$, respectively, in the three scan modes. This variable revisit time optimizes the ability to measure the temporal variability of target signatures caused by apparent changes in temperature and projected area. The angular resolution of $90 \mathrm{mrad}$ relates to the ability to observe individual objects (the classic closely spaced object problem). Since an intercontinental ballistic missile (ICBM) flies for about $30 \mathrm{~min}$, one can envision objects being deployed and separating in a dimension perpendicular to the sensor-to-target line of sight at velocities that differ from one another by $\geq 1 \mathrm{~m} / \mathrm{s}$ for $10 \mathrm{~min}$ before apogee. In such a case, at apogee the apparent object separation of $600 \mathrm{~m}$ is consistent for individual object measurements in the above scenario, since $90 \mathrm{mrad}$ at $6400 \mathrm{~km}$ yields a resolution of about $580 \mathrm{~m}$ for individual pixels of SPIRIT III.

For discrimination purposes, bands A, D, and $E$ have coincident fields of view and record data simultaneously by using dichroic beamsplitters and filters. Such simultaneous measurements eliminate errors due to target motion. Multiple color measurements demonstrate the capability (and limitations) of an infrared sensor to derive important target parameters such as temperature and area. In general, discrimination requires a much higher signal-to-noise ratio (SNR $>20)$ than do detection (SNR $\geq 6$ ) and tracking (SNR $\geq 3$ ). The radiation arriving at a sensor due to thermal emission of a target of area $A$ at range $R$ is $J=$ $\epsilon A L(T) / R^{2}$, where $\epsilon$ is emissivity, $T$ is surface temperature, and $\mathrm{L}$ is the Planck function, which can be integrated for any particular passband. A two-color measurement determines $T$ since the ratio eliminates $R, A$, and $\epsilon$ when $\epsilon$ is the same in both colors. If the range $R$ is known, then $\in A$ can be determined. If the emissivity is less than 1 , then the derivation of area becomes much more complex, since radiation reflected by the body's surface coming from the Earth and the atmosphere must be considered. The multispectral capability of SPIRIT III (bands A, C, D, and $E$ ) will be applied to these cases. For example, the body-reflected radiation in the atmospheric window bands ( $A, C$, and $E$ ) represents integrated surface earth shine, which can be modeled using global satellite scenes and atmospheric sounders to correct for atmospheric transmission and variable emissivities. Earth radiation in band $\mathrm{D}$ emanates from atmospheric $\mathrm{CO}_{2}$ at a colder temperature since it arises from higher altitudes in the mesosphere. The complexities of such analyses must include target shape, surface composition, diffuse vs. specular reflection, emissivity/reflectivity as a function of wavelength, etc., and are the subject of specialized studies beyond the scope of this article.

\section{Space Surveillance Demonstrations}

The MSX satellite is also being used to demonstrate space object surveillance from a space platform, and develop a database (catalog) of Resident Space Object (RSO) observations. The object of space surveillance is to create and maintain a data base on the orbits and signatures of all man-made objects in Earth orbit. RSOs include active payloads, rocket bodies, upper stages, and space debris. The present groundbased Space Surveillance Network (SSN) has limitations in coverage, capacity, sensitivity, available optical wavelengths, and accuracy. MSX will be the first space-based platform to investigate wide-area space surveillance with sensors covering optical wavelengths from the UV to the LWIR.

These catalog maintenance experiments are designed to exploit the greater observing opportunities afforded by a space-based platform

5

American Institute of Aeronautics and Astronautics 
to address the issues of coverage (area of the sky) and capacity (number of objects tracked at one time). These experiments must account for spacecraft constraints, communication limitations, and data accuracy, as well as uncertainties in the existing catalog. Objects to be brought into the catalog come from new launches, lost objects, or breakups. New launches are the most stressing to the SSN due to the requirement to catalog these objects during their first revolution, if possible.

Photometric and radiometric data can also be used in conjunction with high-fidelity satellite models to identify a particular satellite design (e.g. spin stabilized cylinders) and operational status, a process called Space Object Identification (SOI). There are also three experiments concerning space debris. One will use all MSX sensors to develop a multispectral model of space debris. A survey experiment will compile a database of existing debris. The third experiment will capture a satellite breakup, should one occur, and provide detailed data on the resulting debris and its relation to the existing debris population.

The primary MSX sensor for demonstrating space surveillance is the SBV, but the SPIRIT-3 design, though optimized for ballistic missile surveillance, is suitable for demonstrating the added utility of the infrared to this problem.

\section{Background Phenomenology}

A major objective of the MSX mission is to obtain definitive data sets of celestial, earthlimb, and terrestrial backgrounds that could be encountered by operational surveillance sensors. The general objectives of the background measurements are to obtain precise radiometric values in specific optical bandwidths at high spatial resolution to assess the operational limitations that infrared and ultraviolet background structure impose on surveillance systems. These spatial clutter data are supplemented by the spectral sensors which provide critical diagnostic information to understand the spectral content within the broad radiometer bands. The spectral sensors also provide additional data to validate the background models, such as the Strategic High Altitude Radiance Code (SHÄR).

\section{Celestial Backgrounds}

The MSX mission provides unique capabilities to extend the observational database for celestial backgrounds. In the LWIR, the SPIRIT III sensor has about the same sensitivity as the Infrared Astronomy Satellite (IRAS) but about 30 times better spatial resolution. (IRAS was a NASA/UK/Netherlands satellite which mapped $96 \%$ of the sky in four spectral bands from 8 to $120 \mu \mathrm{m}$ in 1983). The SPIRIT III interferometer spectral resolution ranges from comparable to 10 times better. In the ultraviolet, the imagers and spectral imagers will be the most sensitive ever flown for astronomical measurements.

Three celestial backgrounds experiments are designed to complete the LWIR database. They will map: 1) the areas of the sky missed by IRAS; 2) high source density regions, primarily along the galactic plane, where the IRAS survey was confused by multiple sources on a detector; and 3) the sector less than $60^{\circ}$ from the sun. Source lists from these experiments will be combined with the IRAS point source catalog, and their positions improved by identification with stars in astrometric catalogs, to create an all-sky infrared astrometric catalog. LWIR photometry will be done on a number of stars 
for scaling spectral composites or templates which will then be compiled into a stellar atlas to be used for insitu calibration of space-based systems. Asteroids will be observed to ascertain their suitability as calibration sources.

Other experiments include observations in the galactic plane to probe galactic structure; to map extended sources such as $\mathbf{H}$ II (doublyionized hydrogen) regions, large galaxies, and areas of bright infrared structure due to interstellar dust; and to characterize fine scale features in the solar system such as cometary dust trails and the zodiacal dust bands. Interferometry will be done on sources with infrared spectral features (AGB stars and other evolved objects, H II regions, planetary and reflection nebulae) as well as spectral mapping of large bright regions such as the galactic center and the Orion Nebula.

\section{Atmospheric Backgrounds}

Atmospheric background measurements are being collected as a function of tangent altitude, latitude, season, and atmospheric conditions, including quiescent, geomagnetically disturbed, and auroral. The amount and intensity of the structure in a background scene is one of the fundamental limits to the performance of an optical sensor; it produces false targets in the image processor, forcing the tracking processor to deal with many more potential target trajectories than it would otherwise be required to. The goal is to bound the intensity and spectral content of small-scale spatial irregularities of earthlimb ultraviolet, visible, and infrared backgrounds and to determine their global distributions, associations with specific phenomena, and frequencies of occurrence. Earthlimb background experiments will measure the radiance and structure from nadir to full limb viewing geometries at tangent heights up to $300 \mathrm{~km}$.
The atmospheric phenomena to be measured include aurora, airglow, mesospheric clouds, noctilucent clouds, joule-heated atmospheres and stratospheric warmings. Several experiments will be coordinated with ground-based facilities, principally sites for airglow and auroral observations, and space-based assets, such as DMSP and NOAA 11 and 12.

\section{Terrestrial Backgrounds}

Terrestrial backgrounds will be observed episodically by the MSX sensors with particular interest in two wavelength regions where the mean radiance and the spatial structure are reduced due to atmospheric absorption. In the $0.2-0.3 \mu \mathrm{m}$ wavelength region, the middle ultraviolet (MUV), solar albedo is approximately four orders of magnitude less than in the visible regions due to absorption by ozone. Similarly, middle wavelength infrared (MWIR) terrestrial backgrounds are bing observed in two selected bandwidths in the region of strong carbon dioxide absorption at $4.3 \mu \mathrm{m}$. Experiments being executed probe the atmosphere for small-scale structure as a function of altitude. Observations coordinated with corollary assets will provide diagnostics of atmospheric conditions, cloud types, water vapor profiles, aerosol content, and temperature profiles. Corollary sensors include the DOE Atmospheric Radiation Measurement $(\mathrm{ARM})^{s}$ sites, NOAA 11 and 12 satellites, and sites in the National Science Foundation Coupling, Energetics, and Dynamics of Atmospheric Regions (CEDAR) network. ${ }^{6}$

\section{Backgrounds-related SPIRIT-3 Design Issues}

Atmospheric backgrounds play a significant role in the detection and tracking of reentry bodies. Using band A (6-10.9 $\mu \mathrm{m})$ as an exam- 
ple, the dominant atmospheric radiating species is $\mathrm{O}^{3}$ and secondarily $\mathrm{H}_{2} \mathrm{O}$. At the $80-\mathrm{km}$ tangent height of the example above, the integrated nighttime radiance for band $\mathrm{A}$ is approximately $7 \cdot 10^{-7} \mathrm{~W} / \mathrm{cm}^{2}$. This background presents an irradiance at the SPIRIT III aperture of about $2.8 \cdot 10^{-14} \mathrm{~W} / \mathrm{cm}^{2}$ compared with the target irradiance of $1.6 \cdot 10^{-17} \mathrm{~W} / \mathrm{cm}^{2}$ (see the previous discussion). Since this background signal represents a nominal DC radiance that can be filtered by various forms of processors for point source detection, two questions arise: (1) How effective are the processors, and (2) What is the small-scale spatial structure of the background that, after processing, results in false signals (exceedances) above some predetermined threshold and must therefore be handled as "clutter"? If the background structure due to turbulence, gravity waves, etc., can pass through the signal processor at $0.01 \%$ of the DC level, a signal-to-clutter ratio of about 6 would result for the example discussed above. If the "filtered" background structure is $0.1 \%$ of the DC level, then the signal-to-clutter ratio is less than one and target detection and/or tracking becomes more difficult.

Bands B1 and B2, which are located in the middle wavelength infrared (MWIR) spectral region, are selected for their potential to track targets against terrestrial Earth backgrounds. For Earth viewing, the MWIR region is characterized by a very strong $\mathrm{CO}_{2}$ absorption band, which limits the altitude as viewed from space, and therefore clutter, by careful choice of passband. The use of the MWIR region for tracking plumes of high-altitude ICBM stages and post-boost vehicles involves the same Earth-limb and auroral background clutter issue as the other bands. The relevant measure is the transmission to space (nadir viewing) as a function of wavelength. Band B1 (4.22-4.36 $\mu \mathrm{m})$ has essentially zero transmission from below $10 \mathrm{~km}$; therefore, radiance from lower-altitude clouds and the ground is limited. The ground scenes and low-altitude clouds are expected to be more structured than radiance from higher altitudes. Band B2 (4.24-4.45 $\mu \mathrm{m})$ has some transmission down to about $3 \mathrm{~km}(\approx 8 \%$ at the longest wavelength) but does not see to the ground. The overall objective of passband selection is to optimize the target-to-clutter ratio. A nominal theater missile warm body $(350 \mathrm{~K})$ signal can vary by a factor of about 4 over the MWIR region as one widens the long wavelength side from 4.3 to $4.6 \mu \mathrm{m}$. However, the background radiance as seen from space increases by about a factor of $30\left(3 \cdot 10^{-6}\right.$ to $\left.1 \cdot 10^{-4} \mathrm{~W} / \mathrm{cm}^{2} \cdot \mathrm{sr} \cdot \mu \mathrm{m}\right)$, so that the clutter is expected to increase much faster than the target signal as this band is widened to the longer wavelengths. SPIRIT III's mission is to measure globally and categorize by the relevant geophysical parameters for modeling the spatial structure in bands B1 and B2.

Celestial backgrounds (for example, stars, zodiacal light, asteroids, etc.) are also important for surveillance sensors. Although these backgrounds were not considered design drivers, the celestial measurements obtained by MSX are significant and raise some important questions:

- How close to the Sun can an infrared sensor look to maximize coverage? (MSX sensors will measure the near-Sun zodiacal background.)

- Can the infrared stars be used for accurate goniometric purposes? (The infrared centroids will be offset from visible because of dust and gases that surround the star. The MSX sensors will measure the infrared centroids and process the data to achieve measurements on the order of a few microradians.) 
- Can a surveillance sensor discriminate against the celestial background using a stored radiometric catalog and subtraction? (The Infrared Astronomy Satellite [IRAS] catalog is limited to $\approx 45$ stars per square degree, too low for system use. SPIRIT III's resolution is about 30 times that of IRAS. MSX experimental plans are scheduled to map the galactic center to a level of 1100 stars per square degree.)

\section{Sensor Characterization and Calibration}

The careful calibration of the instruments aboard the MSX spacecraft is a critical component in the chain of events from the collection of data to the correct scientific interpretation of those data. The MSX program has recognized this fact by insisting that the data from this experiment meet stringent quality requirements for accuracy and precision.

\section{Sensor Calibrations Use Multiple Approaches}

The calibration of the optical instruments aboard MSX includes both ground testing and on-orbit measurements. The error budget for the calibration has been based on the final specification for the radiometry of the data produced by the MSX sensor suite. The ground chamber testing probes all of the sensor's radiometric and goniometric performance specifications and provides data which directly demonstrate that the instruments meet all of their performance specifications prior to flight. The ground calibration data are also compared to the response of the SPIRIT III sensor to very stable internal sources that provide a link to the on-orbit calibration data and establish the long-term drift in the response as the result of aging of the various optical components. All of the ground test chamber data are traceable to NIST standards.
The on-orbit calibrations use standard reference objects. Stars and other celestial objects are used to verify the ground radiometric and goniometric calibrations. The MSX Program has supported a ground-based, NISTtraceable measurement program which has been designed to measure about 30 reference stars over five years, beginning two years before flight. These stars are the basis for the external calibration of the long- and short-term repeatability of the sensor on-orbit. Because the ground-based observations cannot cover the entire spectrum, due to atmospheric absorption, the relative spectral response calibration are based on not only the reference star photospheric emission models but also on the measurement of six NIST-calibrated reference spheres are be periodically released from the spacecraft. Five black emissive objects will provide secondary infrared calibration sources. One reflective object will validate models of the upwelling earthshine, which is one of the larger uncertainties in the radiometric error budget for the emissive spheres. The spheres are $2 \mathrm{~cm}$ in diameter and are spring-deployed at $13 \mathrm{~m} / \mathrm{s}$.

\section{Characterization of Contamination Effects}

The optical sensors on MSX are designed to strongly reject off-axis radiation, since they will be viewing dim targets in the presence of bright sources such as the earth. Careful baffling and super-polished mirrors can provide excellent performance, but any contamination quickly degrades that performance by an order of magnitude or more. It is important to the complete understanding of the collected data to know how this performance varies with time on orbit. The suite of contamination sensors on MSX are designed to accomplish this; they include a pressure gauge, neutral and ion mass spectrometers, quartz-crystal microbalances 
(with one adjacent to the SPIRIT III main mirror), and flash lamps that work in conjunction with the UVISI instruments to monitor particulates and water vapor in the fields-of-view of the optical sensors. The response of the sensors to internal stimulators and standard stars, as well as their off-axis performance, are monitored over their lifetimes and compared with the contamination data to assess the impact of contamination on the design of operational sensors. These contamination data is also being used to validate the spacecraft contamination control plan and a contamination model that can be used by future spacecraft designers.

\section{Off-axis-rejection Design Issues}

In order to detect and track the small infrared signal of a reentry vehicle as seen from space relatively near the Earth, the design of SPIRIT III had to address another major optical issue: Without the impractical use of an extremely long and cold baffle, radiation from the Earth will strike the primary mirror of the sensor telescope, and the scattered infrared light can be many orders of magnitude larger than the signal. The SPIRIT III solution to this problem was to design and fabricate an off-axis reimaging optical system with super-polished mirrors, Lyot stops and baffles to reduce the amount of diffracted and scattered radiation reaching the focal plane. Ultimate performance depends on how smooth (superpolished) and clean the primary and secondary mirror surfaces are. A technical parameter used as the figure of merit is the bidirectional reflectivity distribution function (BRDF), multiplied by a power of the angular $(\Theta)$ dependence of fall-off of the scattered radiation. ${ }^{7}$

For example, the best measured performance for the pristine-clean and super-polished mirrors of SPIRIT III in the laboratory had a
BRDF of $1 \cdot 10^{-4} \cdot \Theta^{-2}$. The on-orbit performance of SPIRIT III is not yet known, but empirical evidence suggests that the cleanliness has degraded very little on-orbit. The planned measurements of changes in the mirror BRDF with time will provide valuable data for the design of future infrared sensors.

\section{Conclusion}

The MSX satellite has a suite of state-ofthe-art radiometers, imagers, and spectrometers covering the spectrum from the far ultraviolet to the very-longwave infrared. It will not only provide data to answer fundamental questions about the performance of BMDO surveillance systems, but can serve as a highly capable observatory to collect data of broad scientific interest and of potentially high value to the nation. The infrastructure for experiment planning, operations, and data reduction and analysis is in place. It is expected that there will also be many lessons learned in the operation of a tasked spacecraft, distributed data processing, and the efficient archiving of very large data sets that will be of interest to the civilian space community.

Despite the many changes that have occurred in concepts for ballistic missile defense over the period since MSX was designed, it is still the most complete sensor suite for demonstrating the required space functions and obtaining vital phenomenology data. Its primary role is to demonstrate the capabilities of space-based infrared wavelength technology to detect, track, and discriminate ballistic objects and collect the data needed for future system designs. Data to be obtained in the MWIR region $(4.3 \mu \mathrm{m})$ and the medium to long-wavelength infrared region (6-11 $\mu \mathrm{m})$ are particularly applicable to tracking theater-class missiles with large warm bodies. Data in the long-wavelength infrared region (6- 
$16 \mu \mathrm{m}$ ), and particularly in the very-long-wavelength infrared region $(16-28 \mu \mathrm{m})$, are critical for tracking and discriminating the smaller ICBM-class reentry vehicles and decoys. The auxiliary MSX instruments are important for understanding much of the phenomenology associated with the infrared measurements. MSX is the only sensor system of its class and represents a major milestone in the demonstration of ballistic missile defense technology.

\section{Acknowledgments}

The authors particularly wish to acknowledge the early inputs of Barry Katz (SDIO), who is now deceased and is missing the fruits of his labor. A special word of appreciation is due to Major General (Ret.) Garry Schnelzer, formerly of SDIO and the US Air Force, without whose support the project might not have survived the many budget drills. The MSX mission is sponsored by the Ballistic Missile Defense Organization (BMDO). This work was supported under contracts SDIO-8495-0016 and SDIO-84-90-C005.

\section{References}

1. Sharma, R. D., Duff, J. W., Sundberg, R. L., Bernstein, L. S., Gruninger, J. H., Robertson, D. C., and Healey, R J., "Description of SHARC-2, The Strategic High-Altitude Radiance Code," U. S. Air Force Phillips Laboratory, PL-TR-91-2071 (NTIS No. ADA 239008), 1991.

2. Heckathorn, H., and Anding, D., "Strategic Scene Generation Model: Baseline and Operational Software," in Prac. SPIE Conference on the Characterization, Propagation and Simulation of Sources and Backgrounds
III, The International Society for Optical Engineering, Vol. 1967, pp. 151-164, Apr 1993.

3. Mill, J. D., et. al., "Midcourse Space Experiment: Introduction to the Spacecraft, Instruments, and Scientific Objectives," J. Spacecraft and Rockets, 31, (5), 900-907 (Oct 1994).

4. O'Neil, R R., Gardiner, H. A. B., Gibson, J., Humphrey, C. H., Hegblom, R., Fraser, M. E., Kendra, M., Wintersteiner, P., and Rice, C., "Midcourse Space Experiment (MSX): Plans and Capability for the Measurement of Infrared Earthlimb and Terrestrial Backgrounds," in Proc. SPIE International Symposium on Optical Engineering in Aerospace Sensing, The International Society for Optical Engineering, pp. 2223-25, 4-8 Apr 1994.

5. Anon., The Possible Direct Use of Satellite Radiance Measurements by the Atmospheric Radiation Measurement Program, U. S. Department of Energy, DOE/ER-0585T, Mar 1993.

6. Romick, G. J., Killeen, T. L., Torr, D. G., Tinsley, B. A., and Behnke, R. A., "CEDAR: An Aeronomy Initiative," Eos, 68, (2), 19-21 (1987).

7. Uy, O. M., Lesho, J. C., Seiber, B. L., Bryson, R. J., and Wood, B. E., "Optical Effects of Condensates on Cryogenic Mirrors for the Midcourse Space Experiment (MSX)," in Proc. SPIE: Cryogenic Optical Systems and Instruments $V$, Vol. 2227, The International Society for Optical Engineering, pp. 54-63 (1994). 